\title{
Roughening and preroughening in the six-vertex model with an extended range of interaction
}

\author{
Paul J. M. Bastiaansen* and Hubert J. F. Knops \\ Institute for Theoretical Physics, University of Nijmegen, Toernooiveld, 6525 ED Nijmegen, The Netherlands
}

(Received 6 July 1995)

\begin{abstract}
We study the phase diagram of the body-centered solid on solid (BCSOS) model with an extended interaction range using transfer matrix techniques, pertaining to the (100) surface of single-component fcc and bcc crystals. The model shows a $2 \times 2$ reconstructed phase and a disordered flat phase. The deconstruction transition between these phases merges with a Kosterlitz-Thouless line, showing an interplay of Ising and Gaussian degrees of freedom. As in studies of the fully frustrated $X Y$ model, exponents deviating from Ising are found. We conjecture that tricritical Ising behavior may be a possible explanation for the non-Ising exponents found in those models.
\end{abstract}

\section{INTRODUCTION AND MOTIVATION}

The recent interest in surface phase transitions focuses on the interplay between roughening and reconstruction degrees of freedom. ${ }^{1-9}$ The further-than-nearest-neighbor interactions between surface atoms governs the reconstruction of the surface. Den Nijs and Rommelse have established the existence of a phase intermediate between the rough and the reconstructed phase in a simple restricted solid on solid (RSOS) model, in which the surface is disordered but remains flat on average. $^{3}$ They called it the disordered flat (DOF) phase. The principle behind the DOF phase is the simultaneous existence of Ising degrees of freedom (which govern the reconstruction of the surface) and Gaussian degrees of freedom (which govern the roughening), and the possibility of separate and joined transitions of these degrees of freedom.

Recent research on surface models with further-thannearest-neighbor interactions has clarified much of the nature of the DOF phase and its transitions to flat, rough, and reconstructed phases. ${ }^{5,8}$ The long range of the interactions present in these models disables exact solutions, and severely limits the maximum system sizes in numerical calculations. For that reason, only limited work has been done on more realistic models than that studied by Rommelse and Den Nijs. Mazzeo, Carlon, and Van Beijeren studied the (100) surface of a two component bcc crystal like $\mathrm{CsCl},{ }^{9}$ and Mazzeo, Jug, Levi, and Tosatti the (110) surface of a single component fcc crystal, pertaining to the noble metals. ${ }^{6,7}$

The RSOS model of Rommelse and Den Nijs ${ }^{3}$ describes the (100) surface of sc lattices. The reconstructed phase present in their model, which they call body-centered solid on solid (BCSOS) flat, has a simple BCSOS nature and therefore displays an Ising-type degeneracy. These in-plane degrees of freedom become disordered when temperature is increased, giving rise to a DOF phase. It is therefore natural to expect this transition, generally referred to as deconstruction, ${ }^{1}$ to be in the Ising universality class, and indeed this is found in their numerical calculations. The (100) and (110) surfaces of bcc and fcc lattices, on the other hand, give rise to reconstructed phases of a more complicated nature. In the case of a (110) surface this is the missing row (MR) reconstructed phase, also referred to as $2 \times 1$ reconstructed. ${ }^{6}$ In the case of a (100) surface it is the $2 \times 2$ reconstructed phase. ${ }^{9}$ The latter applies to our model. Both phases display a fourfold degeneracy, as will be described in Sec. IV. Deconstruction of this phase can follow different scenarios and there is no a priori reason why its universality class should be Ising. ${ }^{8}$

When the Gaussian, out-of-plane degrees of freedom become disordered, the surface roughens. The roughening transition is of the Kosterlitz-Thouless (KT) type. When both transitions, deconstruction and KT, merge into a single line the surface roughens and deconstructs at the same temperature. The question as to the universality class of this transition seems to have a different answer for different models. Den Nijs ${ }^{5}$ studies the (110) surface of an fcc crystal by means of a four-state chiral clock step model, and finds the transition to be of a decoupled nature, i.e., Ising $\times$ KT. Mazzeo, Carlon, and Van Beijeren, however, find that the two transitions actually never merge but only become exponentially close. ${ }^{9}$ Nevertheless, the exponents on the deconstruction branch deviate from Ising even when both transitions are still well separated. ${ }^{9,10}$ We shall come back later to this point.

The close interplay between Ising and Gaussian degrees of freedom is also observed in fully frustrated $X Y$ models $(\mathrm{FF} X Y)$, where the frustration is responsible for an Isingtype degeneracy, whereas the $X Y$ degrees of freedom are Gaussian. ${ }^{11-17}$ The generic version of this type of models, the coupled $X Y$-Ising model, ${ }^{12,18}$ is actually dual to the clock step model of Den $\mathrm{Nijs}^{5}$ in the zero chirality limit. In the FFXY models, both transitions are found to be either closely separated $^{17}$ or simultaneous, and exponents deviating from Ising are found by many authors. The same puzzling phenomenon thus is observed here, and the question as to the universality class of the transition in the FFXY models may well be the same question as in the case of the surface models.

In this paper, we present the study of the (100) surface of a single component fcc crystal like argon. The model is equipped with further than nearest neighbor interactions, and we believe it to be a realistic description of these surfaces. In another paper, we present Monte Carlo (MC) simulations on a Lennard-Jones fcc structure pertaining to argon, to calculate the coupling constants of our model. ${ }^{19}$ Our model exhibits a $2 \times 2$ reconstructed phase which is not, to our knowl- 


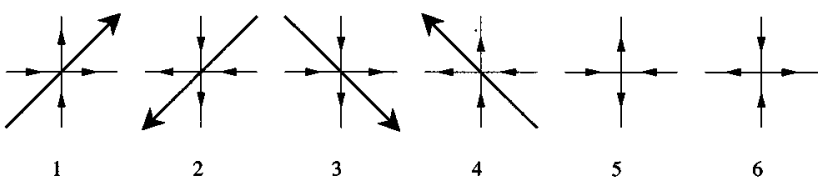

FIG. 1. The vertices of the six-vertex model. Height differences on the sites are indicated with thin arrows. The bold arrows indicate the net polarization of the vertices, and correspond to a height difference on one of the two sublattices. The vertices 5 and 6 are flat and remain empty.

edge, realized in nature. Indeed, our MC calculations on argon show that its (100) surface does not exhibit a DOF or reconstructed phase. Nevertheless, our calculations indicate what kind of interactions on a (100) surface can give rise to DOF phases.

Moreover, we also observe that the interplay between roughening and Ising degrees of freedom yields exponents deviating from Ising. We find that the exponents agree fairly well with those found in the $\mathrm{FFX} Y$ models, and argue that both transitions are in the same universality class. To classify the values of the exponents at the deconstruction transition, we want to put forward the conjecture that instead of Ising behavior, tricritical Ising behavior may be involved.

This paper is organized as follows. In Sec. II we give a description of our model. In Sec. III we present the phase diagram. In Sec. IV we study the ordered phases and the possible interfaces between them, in order to understand qualitatively the behavior of the model and to present the techniques used to derive the phase diagram. In Sec. V we present the results of the calculation of the critical exponents. In Sec. VI we put forward our conjecture of tricritical Ising behavior.

\section{DESCRIPTION OF THE MODEL}

The model under study is an extended version of the $F$ model $^{20}$ which was exactly solved by Lieb. ${ }^{21}$ The $F$ model falls into the larger class of six-vertex models. In 1977, Van Beijeren formulated these six-vertex models in terms of heights, in order to use them to describe surfaces of bcc and fcc crystals. ${ }^{22}$ Hence the name BCSOS model.

Our model is formulated on a square lattice, where on every lattice site a height variable is defined that can have integer values, with the restriction that nearest neighbor heights always differ by +1 or -1 . This can be represented by putting an arrow on each bond of the dual lattice, giving rise to the six possible vertices of the six-vertex model on the sites of the dual lattice. The arrows of each vertex satisfy the ice rule: two arrows point inward, two arrows point outward (Fig. 1).

The formulation in terms of heights gives rise to two equivalent sublattices, with heights even on one lattice and odd on the other. Let us, throughout this paper, denote the "even" sublattice with $A$ and the "odd" sublattice with $B$. The interactions between heights of different sublattices only exists by means of the above-mentioned restriction, whereas the interaction between heights of the same sublattice are between nearest and next-nearest neighbors. Equal heights of the atoms are given a Boltzmann weight 1 , whereas height

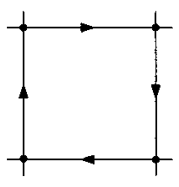

1

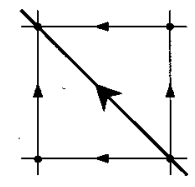

$\mathrm{WK}^{2}$

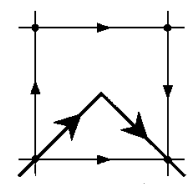

WK

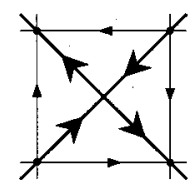

$\mathrm{w}^{2}$
FIG. 2. Boltzmann weights in terms of loop configurations. Dots denote the positions of the vertices. The presence of a loop (thick lines) between two atoms of one sublattice denotes a height difference of these atoms. Loops are characterized as $A$ or $B$ loops, depending on the corresponding sublattice.

differences of \pm 2 are given a Boltzmann weight $W$ in the case of nearest neighbors and $K$ in the case of next-nearest neighbors. $W$ and $K$ are the parameters of the phase diagram. We limit ourselves to that part of the phase diagram where inequality of next-nearest neighbor heights is disapproved of, in other words, $K \leqslant 1$.

An alternative formulation is obtained when height differences between atoms of a sublattice are indicated by an oriented loop. Each vertex corresponding to a height difference on one of the two sublattices carries a net polarization that can be indicated by an arrow, as depicted in Fig. 1. The collection of all polarization arrows forms loops, each loop pertaining to one of the two sublattices, so that we can distinguish between $A$ and $B$ loops. A loop indicates a height difference of \pm 2 . Adjacent loops of the same sublattice carry antiparallel arrows, whereas adjacent loops of different sublattices carry parallel arrows. Loops of different sublattices do not cross. In terms of these loops, the Boltzmann weights are $W K^{2}$ per unit length for a straight piece, $W K$ for a corner and $W^{2}$ for an intersection; see Fig. 2.

The line $K=1$ in the phase diagram implies absence of next-nearest-neighbor interactions, and the model equals the exactly solved $F$ model. On this line, the surface is flat for $W<\frac{1}{2}$, and exhibits a KT transition at $W=\frac{1}{2}$ to a rough phase. We check our computer program against this exact solution, and it enables us to get an indication of the accuracy of the techniques used to estimate the critical exponents of the model. This is the more important, as the long range of the interaction limits the maximum system size which we can reach with our calculations.

The other extreme of the phase diagram is $K=0$, where all height differences between next nearest neighbors of a sublattice are forbidden. The line $K=0$ thus corresponds to a flat surface for all $W$. This can be established by filling the lattice with vertices 5 and 6 in a checkerboard configuration. No loops are present on the lattice, and its free energy is equal to 0 . This phase is twofold degenerate, and exists for $W<1$. The average height $\langle h\rangle$ is half-integer.

For $W>1$, it is cheaper to form a reconstructed phase, where the heights of one of the two sublattices are all equal (say 0 ), whereas the heights of the other sublattice are alternately +1 and -1 . In terms of loops the lattice is filled with intersections, as in Fig. 3, where the phase is depicted, together with possible interfaces between the different realizations of this phase. $A(B)$ loops indicate height differences of the even (odd) sublattice and are depicted as solid (dashed) lines. The phase is called $2 \times 2$ reconstructed. It has a free 


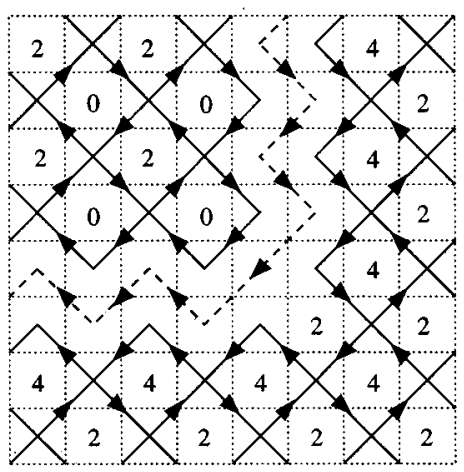

(a)

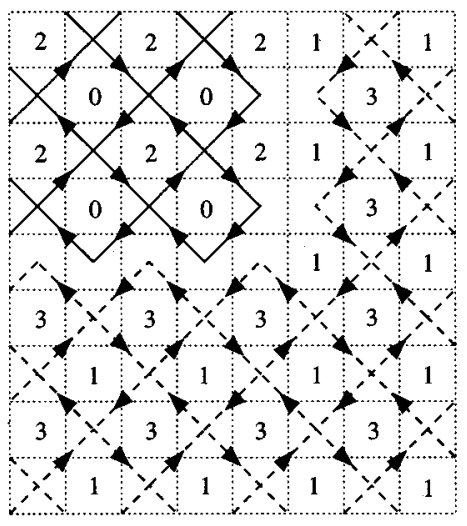

(c)

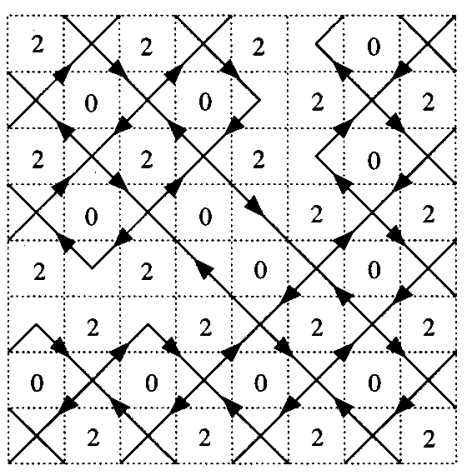

(b)

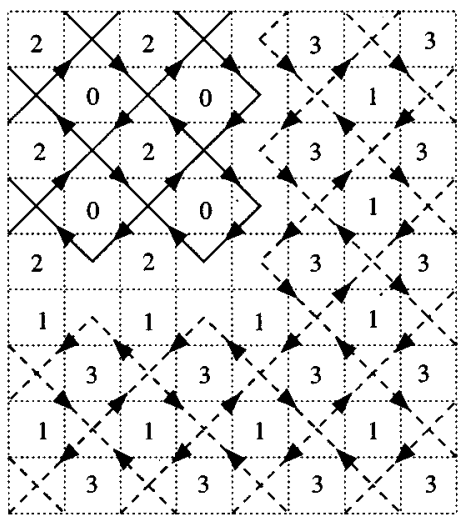

FIG. 3. Interfaces between different realizations of the $2 \times 2$ reconstructed ground state. The configuration is depicted in terms of the loops in Fig. 2. Vertices reside on the sites of the square lattice (thin, dotted lines). The solid lines are the $A$ loops; the dashed lines are the $B$ loops. Digits indicate the heights of the alternating sublattice; heights of the fixed sublattice not being indicated. The phase in the upper left corner of each of the pictures is $A^{+}(1)$, the 1 indicating the average height. (a) is the double step interface between $A^{+}(1)$ and $A^{+}(3)$, (b) is the Ising interface between $A^{+}(1)$ and $A^{-}(1)$, and (c) and (d) are the single step interfaces between $A^{+}(1)$ and $B^{+}(2)$ and $B^{-}(2)$, respectively. The "thick" part of the $A-B$ interface can be seen as a "thin" part together with an additional Ising interface. energy $f=-\ln (W)$ for $K=0$, and will therefore be stable for $W>1$. It is fourfold degenerate (apart from the infinite degeneracy resulting from overall height changes). At $W=1$ there is a phase transition between the flat and the reconstructed phase. At and slightly above $K=0$ all possible excitations are heavily suppressed, so that the transition must be of first order.

\section{THE PHASE DIAGRAM}

First let us present the phase diagram of the model (Fig. 4). The line $K=1$ corresponds to the exactly solved $F$ model. For $W<\frac{1}{2}$ the surface is flat. At $W=\frac{1}{2}$ there is a $\mathrm{KT}$ transition into the rough phase, where the model renormalizes to the Gaussian model. The whole area below the line $T-R-S-U$ belongs to this rough phase.

At $K=0$, where next nearest neighbors at both sublattices are forced to be of equal height, there is a first order transition from the flat phase into the $2 \times 2$ reconstructed flat phase. At point $Q$ this first order transition goes over into the line $Q-R$, which is a preroughening line. ${ }^{3}$ To the right of this line there is a DOF phase. The line $Q-S$ is an Ising-like transition into the $2 \times 2$ reconstructed phase. We do, actually, not find Ising exponents on this line.

This part of the phase diagram closely resembles that of Den Nijs. ${ }^{4}$ In this reference he considers an RSOS model with nearest and next-nearest-neighbor interactions. He also finds a first order line, where the average surface height changes from integer to half integer, continuing into a preroughening line.

At point $Q$ in our phase diagram, the interface free energy between integer surface height and half-integer surface

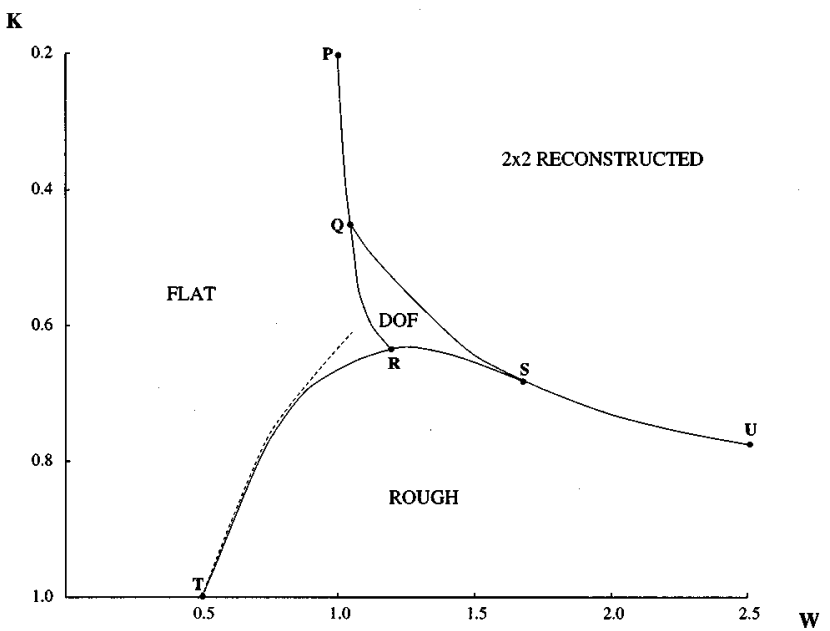

FIG. 4. The phase diagram of the BCSOS model with extended interaction range. The parameter $W$ is the Boltzmann weight pertaining to a nearest neighbor height difference on a sublattice. $K$ is the weight pertaining to a next nearest neighbor height difference. The line $K=1$ corresponds to the exactly solved $F$ model. The dashed line is the estimate $W K^{3 / 2}=\frac{1}{2}$ of the KT transition. 
height vanishes, which corresponds to $\frac{1}{2}$-step melting. On the preroughening line the surface is rough.

On the line $S-U$ the surface roughens and deconstructs simultaneously. In this respect our phase diagram differs from that of Ref. 4 where the two transition lines (roughening and deconstruction) actually cross. The behavior in our model is more similar to the clock step model analyzed later by Den Nijs, ${ }^{5}$ where the reconstructed rough phase does not exist. This phase, where the Ising order is still present but the surface is already rough, does not exist in our model. The transition line $S-U$ therefore includes roughening and simultaneous disordering of the Ising degrees of freedom. We do not find Ising exponents on this line.

The merging of the Ising-like transition $Q-S$ and the KT line $R-S$ is also described by Mazzeo et al. ${ }^{9}$ They claim that in their model the lines never actually merge, but become more and more closely separated. We believe, however, that this question is by no means settled and cannot be settled by purely numerical methods. Two separate transitions occurring nearby will strongly influence each other and are likely to join. A true resolution of this point will require analytical methods, probably employing the supersymmetry which might be invoked by $S T$ invariance. ${ }^{5}$

In our calculations, the limited system size prevents an accurate determination of the transition points. We choose to interpret our data such that both lines meet at point $S$, but stress that we are neither certain of its existence nor of its precise location. An analytical approach will be necessary to settle this open question.

\section{INTERFACES AND CRITICAL EXPONENTS}

In both the flat and the reconstructed phase the surface is ordered. The flat phase is twofold degenerate with respect to its arrow representation, the phases corresponding to an average height of $\pm \frac{1}{2} \bmod 2 n$, respectively. It undergoes a roughening transition when the free energy of the interface between the two realizations of this phase vanishes. This interface has the character of a single step and consists of the $A$ or $B$ loops in Fig. 2 as discussed before. At $K=1$ this transition takes place at $W=\frac{1}{2}$. The weight per unit length of the interface is then $W$. For $K \neq 1$ this weight is $W K^{2}$ for a straight piece and $W K$ for a corner. The average weight is therefore $W K^{3 / 2}$ and we can estimate the KT line $T-R$ by plotting $W K^{3 / 2}=\frac{1}{2}$ in the phase diagram (the dotted line in Fig. 4), giving a good agreement with the actual line. This means that roughening on the line $T-R$ is established via the same mechanism as in the $F$ model.

The reconstructed phase is fourfold degenerate. Heights on one sublattice are fixed, whereas heights on the other sublattice alternate in a checkerboard fashion, which can be chosen in two equivalent ways. The average height equals an integer. The ground state itself corresponds to a lattice filled exclusively with intersections of one type of loop. This is depicted in Fig. 3, together with possible interfaces between the different realizations of this phase. Note that the degeneracy present in the MR reconstructed phase of (110) surfaces is of the same type.

Let us denote the phases by $A^{+}(n), A^{-}(n), B^{+}(n)$, and $B^{-}(n)$. The $A$ and $B$ refer to the loop type. For the phase $A^{ \pm}(n)$, the heights of sublattice $A$ alternate between $n+1$

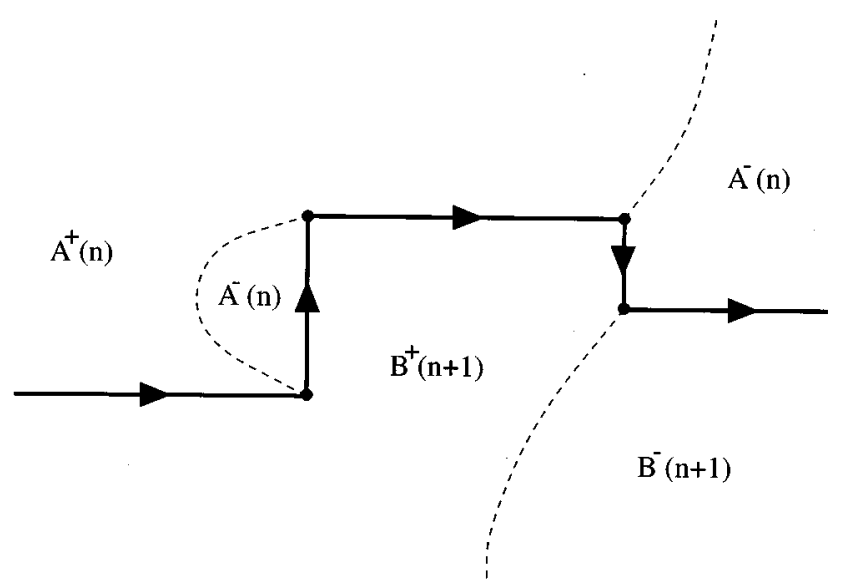

FIG. 5. The behavior of the $A-B$ interface of Figs. 3(c) and 3(d). The "thick" part of the depicted interface consists of a "thin" part and an Ising part, which can split off and wander over the lattice, eventually become connected to other single step interfaces. The solid line is a pure $A-B$ interface; the dashed lines are Ising walls.

and $n-1$ and those of sublattice $B$ are fixed. The plus and minus signs refer to the antiferromagnetic order.

The integer $n$ is the height of the fixed sublattice, which is equal to the average height of the phase. We thus have the following phases:

$$
\begin{aligned}
& A^{+}(n) \text { and } A^{-}(n) \text { with } n \text { odd, } \\
& B^{+}(n) \text { and } B^{-}(n) \text { with } n \text { even. }
\end{aligned}
$$

It follows that an interface between $A$ and $B$ always carries (at least) one step up or down.

Figure 3 shows four possible interfaces, bending around a corner. The phase in the upper left corner in each of the four figures is $A^{+}(n=1)$. The interface in Fig. 3(a) is between $A^{+}(1)$ and $A^{+}(3)$, and does therefore not affect the Ising order, but only the roughness of the surface. It carries a double step. The interface in Fig. 3(b) is between $A^{+}(1)$ and $A^{-}$(1) and does not carry a step but has the character of a pure Ising-Bloch wall.

In Figs. 3(c) and 3(d) the interfaces are between $A^{+}(1)$ on the one hand and $B^{+}(2)$ and $B^{-}(2)$ on the other. As can be seen from the figure, the character of the interface is different in the horizontal and vertical directions. The thin, "cheap" part of the interface can be seen as a pure $A-B$ interface, carrying a single step, whereas the thick, "expensive" part is an $A-B$ interface together with an Ising wall, which, in the figure, is depicted alongside the single step interface. A corner in the $A-B$ interface thus creates an Ising wall, which can eventually split off and wander freely over the lattice, thereby gaining entropy, as depicted in Fig. 5. It follows that there is an important interplay between the interfaces of Figs. 3(b), 3(c), and 3(d), or between roughening and reconstruction degrees of freedom. An attempt to locate the transition points by just estimating the interface free energies is therefore likely to fail.

Now it can be seen that in principle two different scenarios for deconstruction can be imagined. ${ }^{8}$ When only the free energy of the pure Ising interface vanishes, the Ising order is destroyed. The surface remains flat, as the domain 
wall does not carry a step, and only one type of loop intersections $(A$ or $B)$ prevails on the surface. It is natural to assume that this scenario for deconstruction falls in the Ising universality class. The second scenario applies when single step interfaces become free, but steps occur in an up-downup-down order, such that the surface remains flat. This scenario can apply when parallel steps are repulsive and antiparallel steps are attractive. This is the qualitative mechanism as described by Rommelse and Den Nijs. ${ }^{3}$ In this case the Ising order is destroyed and both loop types $A$ and $B$ appear on the surface. There is no a priori reason for this deconstruction scenario to fall in the Ising universality class. Instead, one could argue it to be prerougheninglike as the transition involves two phases with different average heights merging into a single phase with an intermediate average height. See also Ref. 8 and Sec. V.

The two scenarios give rise to different DOF phases. In the first scenario, the DOF phase is actually ordered with respect to the prevailing sublattice loops $A$ or $B$ and is therefore called deconstructed, even, flat (DEF) by Bernasconi and Tosatti. ${ }^{8}$ The second scenario gives rise to sublattice as well as Ising disorder. We will see that in our model the first scenario applies; therefore the appearing phase is actually $\mathrm{DEF}$, but as both types generally are referred to as DOF in the literature, we chose to follow this convention.

A method to extract information on the phase diagram and critical exponents is to force the system to generate interfaces by applying different boundary conditions (BC's). When the ground state does, as a result of the BC's, not fit on the lattice, the system will be forced to generate an interface at the expense of a higher free energy. Subtracting the free energy of the system without an interface yields the pure interface free energy $\eta$. To calculate these free energies we employ transfer matrix calculations on lattices of dimension $L \times \infty$. As compared to Fig. 3, we choose the direction of transfer to be diagonal. The original six-vertex square lattice is then tilted over 45 degrees. This enables us to do calculations on lattices with a maximal dimension of $10 \sqrt{2}$ in terms of the lattice distance. We denote this dimension by $L=10$. The interface free energy $\eta(L)$ per unit length is then calculated as

$$
\eta(L)=-\frac{1}{L} \ln \left(\frac{\lambda^{\prime}}{\lambda}\right)
$$

where $\lambda$ is the largest eigenvalue of the transfer matrix with $L$ even and periodic BC's and $\lambda^{\prime}$ is the largest eigenvalue pertaining to other BC's. For $L$ odd, we interpolate between $L-1$ and $L+1$.

With periodic BC's, the net number of steps on the lattice is a conserved quantity. As a result, the transfer matrix splits up into blocks, each block corresponding to a number of steps which is $0, \pm 2, \pm 4, \ldots .{ }^{23}$ The ground state, or the largest eigenvalue, is to be found in the central block of the transfer matrix, corresponding to a net number of zero steps on the surface. We also calculate the largest eigenvalue in the subcentral block corresponding to two up or down steps. The corresponding interface free energy is denoted by $\eta_{s}(L)(\eta$ step).

It is readily seen that the reconstructed ground state only fits on the lattice when $L$ is even and periodic BC's are applied. When $L$ is odd the ground state only fits over the cylinder when it is shifted by one unit in the diagonal direction. Under such a shift $A^{ \pm}(n)$ turns into $A^{\mp}(n)$ (and $B^{ \pm}$ into $\left.B^{\mp}\right)$; hence the system is forced to generate an Ising wall. The corresponding interface free energy will be denoted by $\eta_{o}(L)$ ( $\eta$ odd).

Furthermore, we perform calculations with antiperiodic BC's. The arrows on the bonds of the six-vertex lattice are flipped on the boundary. As a result, the net number of steps on the surface is not conserved anymore. Antiperiodic BC's also generate an Ising wall, but imply in addition an inversion $h \rightarrow-h$ of the Gaussian height variables. The interface free energy will be denoted by $\eta_{-}(L)$.

And finally, we calculate the largest eigenvalue in the central block of the transfer matrix that corresponds to an eigenvector which is antisymmetric under arrow inversion. This eigenvalue allows us to calculate an inverse correlation length which will be denoted by $\eta_{i}$ ( $\eta$ Ising),

$$
\eta_{i}(L)=-\frac{1}{L} \ln \left(\frac{\lambda_{\mathrm{as}}}{\lambda_{s}}\right),
$$

where $\lambda_{\text {as }}$ is the largest eigenvalue corresponding to an eigenvector which is antisymmetric under arrow inversion. $\lambda_{s}$ is the maximum eigenvalue of the transfer matrix, whose eigenvector is symmetric under arrow reversion. Hence the subscripts. The correlation length corresponds to the Ising order as follows from the symmetry of the involved eigenvector.

We do, actually, need to generate still two additional interfaces. We need to distinguish between the two different deconstruction scenarios described above. Therefore we need to decide whether there is either only Ising disorder or Ising as well as sublattice disorder in the DOF region. The required BC's therefore should (a) generate an odd number of steps on the surface, thus coupling an $A$ and a $B$ phase over the boundary, and (b) be antiperiodic in order to retain the up-down-up-down order of steps (there can be no up-downup-down order of steps when the number of steps is odd and periodic BC's are applied). The corresponding interface free energy then vanishes when there is sublattice disorder but remains finite when there is only Ising disorder in the DOF region. The second additional interface we need to generate is the pure single step interface which we need to confirm the Gaussian nature of the preroughening line.

With the direction of transfer chosen as above, it is impossible to generate these two interfaces. Therefore we perform limited calculations on the same model, but with the direction of transfer chosen as the vertical direction with respect to the lattice depicted in Fig. 3. The ground state then fits on the lattice when its size is an even number of vertices and when periodic BC's are applied. With respect to this direction, a vertical unit shift turns $A^{ \pm}$into $B^{ \pm}$(and a horizontal unit shift turns $A^{ \pm}$into $B^{\mp}$ ). Hence a single step interface can be generated by choosing the system size odd. The interface decisive of sublattice disorder is generated by choosing the system size odd and applying antiperiodic BC's as well.

The interface free energies allow us to distinguish between the various possible phases. Extrapolating $L \rightarrow \infty$ yields the infinite size free energy. Performing the extrapola- 
tion with and without the value for the lowest row size $L=2$ yields an estimate of the error. In the flat phase, only $\eta_{o}$ vanishes. In the reconstructed flat phase $\eta_{o}, \eta_{-}$, and $\eta_{s}$ are finite. In the DOF phase, $\eta_{-}$and $\eta_{o}$ vanish, and in the rough phase all interface free energies vanish. Vanishing interface free energies all exhibit an exponential finite size dependence everywhere but at criticality, where they scale as $1 / L$. Plotting $L \eta(L)$ for various system sizes thus yields information about phases, phase transitions, and critical exponents by standard techniques of finite size scaling (FSS). ${ }^{24}$

Interface free energies are inverse correlation lengths, and scale as

$$
L \eta(L)=\frac{L}{\xi} \rightarrow 2 \pi x
$$

at criticality, where $x$ is the critical exponent pertaining to the correlation function of the disorder operator that generates the interface in question. ${ }^{25}$ The exponent $x$ is extracted by plotting $L \eta(L)$ for different values of the system size $L$, and extrapolating the values at the intersection points of the curves. The central charge can be calculated from the finite size dependence of the free energy ${ }^{26}$

$$
f(L)=f(\infty)-\frac{\pi c}{6 L^{2}}
$$

The double step interface $\eta_{s}$ scales in the rough phase as

$$
L \eta_{s}(L)=\frac{1}{2} K_{g} a^{2},
$$

where $K_{g}$ is the value of the Gaussian coupling and $a=2$ is the step height. We consider double steps, and extract $K_{g}$ by extrapolating $\frac{1}{2} L \eta_{s}(L)$. Single steps are more difficult to treat in this model, as they couple to the Ising order as well. We will only occasionally need those single step interfaces, to distinguish between the two possible deconstruction scenarios giving rise to DOF and DEF phases, respectively, and to establish the Gaussian (rough) nature of the preroughening line. When the model renormalizes to the Gaussian model, the ratio of the single and double step free energy is precisely 4 , as can be read off from Eq. (6). We will use this prediction to confirm the Gaussian nature of the preroughening line.

In the rough phase close to the KT transition, $K_{g}$ assumes the behavior ${ }^{27}$

$$
K_{g}=\frac{1}{2} \pi+A \sqrt{T-T_{c}},
$$

where the critical value $\frac{1}{2} \pi$ and the square root are universal. The quantity $\left(K_{g}-\pi / 2\right)^{2}$ should vanish linearly when approaching a KT point. We use this linear behavior as the identification of a KT transition.

Antiperiodic BC's imply in particular an inversion $h \rightarrow-h$ of the Gaussian height variables. In the rough phase and on the KT lines, where the model renormalizes to the Gaussian model, this inversion yields a universal defect free energy $^{28}$

$$
L \eta_{-}(L) \rightarrow \frac{1}{4} \pi
$$

independent of the value of the Gaussian coupling $K_{g}$. In the present model on the line $S-U$ in the phase diagram, where Gaussian degrees of freedom couple to the deconstruction degrees of freedom, it is not a priori clear how to disentangle this contribution. However, in order to see whether in the scaling limit a decoupling scenario makes sense, it will be useful to simply subtract this contribution from the value of $L \eta_{-}(L)$.

It is often taken for granted that the interface free energies $\eta_{o}$ and the inverse correlation length $\eta_{i}$ must yield the same exponent $x$. The BC's used to calculate $\eta_{o}$ generate an Ising wall and correspond to the correlation function of a disorder operator, which is, in the Ising model, dual to the spin-spin correlation function. $^{29}$ This is not necessarily true in the present case (cf. also Ref. 16), and we will carefully distinguish the different exponents by indicating them with $x_{o}$ and $x_{i}$, respectively. The exponent from $\eta_{-}$will be indicated as $x_{-}$. The exponent pertaining to $\eta_{s}$ is involved when roughening takes place and is conventionally expressed in terms of the Gaussian coupling $K_{g}$. The thermal exponent $x_{t}$ is calculated from the singular behavior of the specific heat. The singular part of the specific heat $C$ is in our model proportional to the variance of the number of broken (next) nearest neighbor bonds:

$$
C \sim-W \frac{\partial}{\partial W}\left(W \frac{\partial}{\partial W} f(W, K, L)\right),
$$

or a similar expression with derivatives with respect to $K$. The specific heat scales as

$$
C \sim L^{2-2 x_{t}}
$$

at criticality, ${ }^{24}$ enabling us to extract the value of $x_{t}$.

\section{RESULTS}

\section{A. The KT and preroughening lines}

In the rough phase, under the line $T-R-S-U$, the model renormalizes to the Gaussian model. $L \eta_{-}(L)$ assumes its universal value $\frac{1}{4} \pi$ as it should [Eq. (8)]. The line $T-R-S$ is identified as a KT transition, where single steps melt, via the linear behavior of $\left(K_{g}-\frac{1}{2} \pi\right)^{2}$ as in Eq. (7).

The line $P-Q$ is a first order transition and goes over into the preroughening line $Q-R$. At the preroughening line, the surface is rough and the model renormalizes to the Gaussian model. The preroughening transition, together with its first order continuation, is now well understood. ${ }^{3,5}$ It draws a close resemblance to the $F$ line which is, in our phase diagram, the line $K=1$. At the first order line, there is coexistence of different ordered phases with different surface heights. At point $Q$ (or similarly, at point $T$ ), the interface free energy between these different heights vanishes, and the surface roughens. Coexistent phases at point $Q$ are integer valued (the reconstructed phases) and half-integer valued (the flat phases). This means that roughening of the surface takes place via $\frac{1}{2}$-step melting. As a consequence, the universal value of the Gaussian coupling $K_{g}$ equals $2 \pi$ at point $Q$. The preroughening line $Q-R$ is a line with continuously varying critical exponents, as is the $F$ line for $W>\frac{1}{2}$.

Roughening of surfaces is conventionally described by the sine-Gordon Hamiltonian 
L $\eta_{-}(\mathrm{L})$

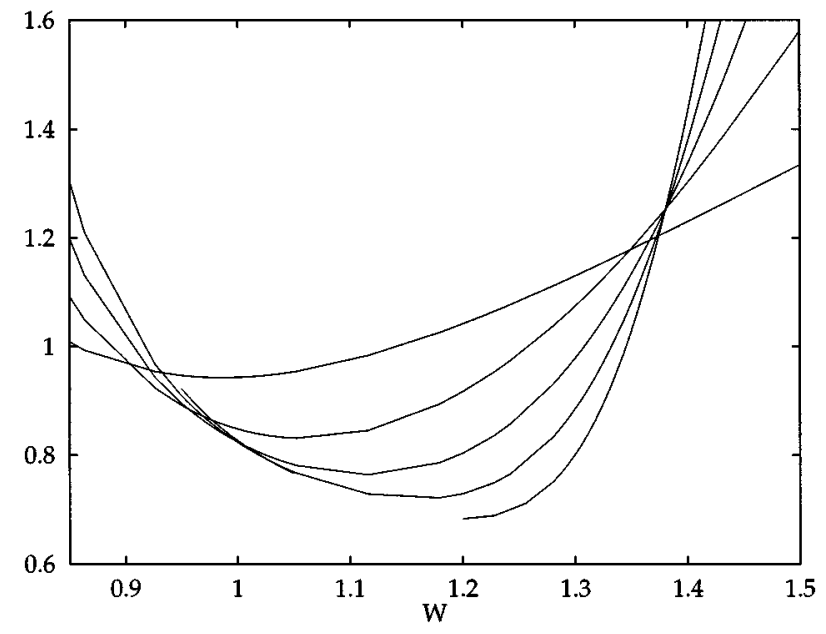

FIG. 6. Seam free energy $L \eta_{-}(L)$ for $L=2,4,6,8,10$ on the line $K=0.60$. Larger $L$ values correspond to steeper curves. The intersection points on the right and on the left clearly correspond to different locations. The values of $L \eta_{-}(L)$ in between drops to zero, indicating a DOF phase. The intersections on the left converge to $\frac{1}{4} \pi$, indicating the Gaussian character of the preroughening line. The intersection points on the right clearly are above $\frac{1}{4} \pi$, indicating the non-Ising character of the deconstruction transition.

$$
\begin{aligned}
H= & \int d^{2} r\left\{\frac{1}{2} K_{g}\left|\nabla \phi_{\mathbf{r}}\right|^{2}-U_{2} \cos \left(2 \pi \phi_{\mathbf{r}}\right)\right. \\
& \left.-U_{4} \cos \left(4 \pi \phi_{\mathbf{r}}\right)\right\},
\end{aligned}
$$

where $\phi$ denotes the average surface height. In the flat phase, the average surface height is half-integer, which means that $U_{2}<0$. In the reconstructed phase this average height is integer, meaning $U_{2}>0$. The line $Q-R$ therefore must correspond to $U_{2}=0$, meaning that integer as well as half-integer average surface heights are allowed. The renormalization towards the Gaussian model on this line is governed by the parameter $U_{4}$ which remains irrelevant up to the point $Q$ where $K_{g}$ takes the renormalized value $2 \pi$.

Our numerical calculations confirm this. On the preroughening line $L \eta_{-}(L)$ converges to $\frac{1}{4} \pi$ as it should. The value of $K_{g}$ should equal $\frac{1}{2} \pi$ at point $R$ and increase to $2 \pi$ at point $Q$. We find at $K=0.60$ the value $K_{g}=1.754(14)$, slightly above $\frac{1}{2} \pi$, and $K_{g}=2.07(5)$ at $K=0.55$. $K_{g}$ increases further to $2 \pi$ at point $Q$. Moreover, Gaussian behavior predicts that the ratio of the single and double step interface free energies is 4 . We determine this ratio at point $K=0.55$ and find it to be $4.1(2)$.

The DOF phase, confined by the lines $Q-R-S-Q$, is characterized by a finite value of the double step interface free energy and vanishing of $\eta_{o}$ and $\eta_{-}$. Also the central charge $c$ should converge to zero in this region, but we do not see this as there is a strong crossover to Gaussian behavior in this region. Clear evidence for the existence of the DOF phase is given in Fig. 6, where $L \eta_{-}(L)$ is plotted for different values of $L$ on the line $K=0.60$. Intersection points of the curves indicate critical points. Two clearly distinct inter-

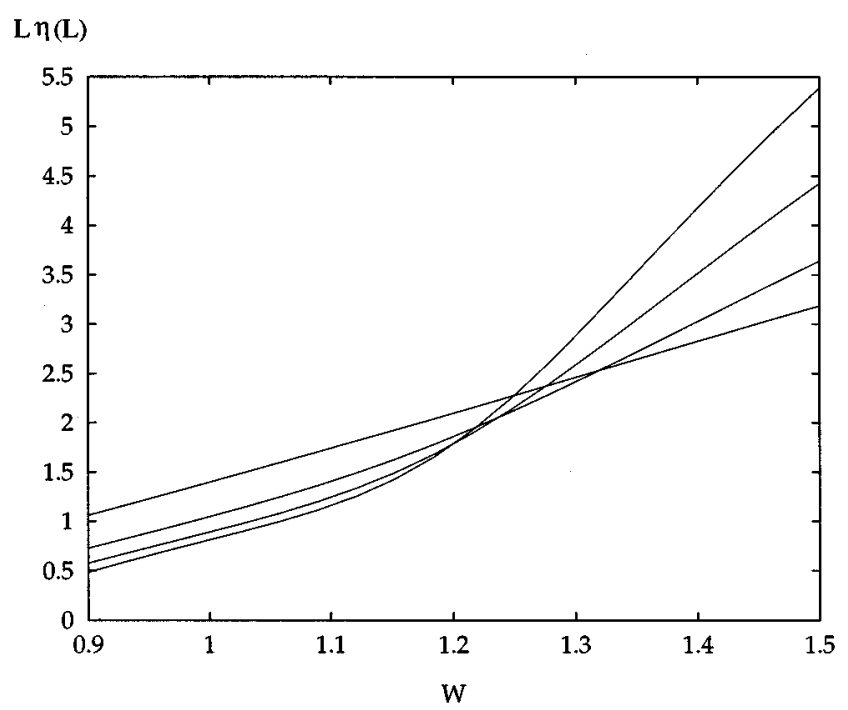

FIG. 7. The interface free energy $L \eta(L)$ of the interface which distinguishes between sublattice order and disorder in the DOF region. It is calculated using antiperiodic boundary conditions that couple $A$ loops with $B$ loops over the boundary. It is calculated on the line $K=0.55$ for $L=5,7,9,11$, curves increasing on the right corresponding to increasing values of $L$. Extrapolating the location of the intersection points gives a value of $W \approx 1.03$. The preroughening transition is found to be at $W=1.08$ on this line, whereas deconstruction is located at $W=1.25$. This implies that the intersection points actually belong to the preroughening transition, and that $\eta(L)$ remains finite in the DOF region, indicating sublattice ordering. See the text for further explanations.

section points are found on this line, the value of $L \eta_{-}(L)$ strongly decreases in between these points, and we expect it to drop to zero for larger values of $L$. We take this as conclusive evidence for the existence of a DOF phase in between these points.

Strong crossover is to be expected in the DOF region, which is relatively small, and on the line $Q-S$, and we should be careful interpreting our data. The parameter $U_{2}$ of the sine-Gordon model in Eq. (11) is relevant in the renormalization sense, but still small, as it vanishes on the preroughening line. From the line $R-S$ we see that the value of the Gaussian coupling $K_{g}$ is indeed above its universal value $\frac{1}{2} \pi$, but yet slightly. This means that the DOF region, together with the line $Q-S$, exhibits a strong Gaussian-like behavior and that the real, flat nature of the surface only becomes apparent for much larger system sizes.

\section{B. The line $Q-S$}

The most interesting part of the phase diagram are the lines $Q-S$ and $S-U$, as they exhibit the interplay between roughening and reconstruction degrees of freedom. The location of the line $Q-S$ is determined by the vanishing of the interface free energies $\eta_{-}$and $\eta_{o}$. First we determine which of the two scenarios, as described in Sec. IV, applies to the deconstruction transition $Q-S$. We examine the behavior of the required interface as described in this section. It is calculated using the "vertical" transfer matrix, odd system size, and antiperiodic BC's. Its free energy on the line $K=0.55$ is 
depicted in Fig. 7. We find that it remains finite in the DOF region up to the preroughening line. This is conclusive to decide that it is the first scenario which applies, meaning that only the Ising order is destroyed in the DOF region, but that still one of the two sublattice loops $A$ or $B$ prevails on the surface. It is therefore expected that the line $Q-S$ is an Ising transition with central charge $c=\frac{1}{2}$. We are, however, not able to confirm this.

On the line we find, as expected, strong crossover to Gaussian behavior. Convergence of the central charge and the exponent pertaining to $\eta_{-}$is not smooth. Of the central charge, no estimate whatsoever is made. The exponent $x_{-}$ varies from $0.173(9)$ to $0.192(5)$ in the direction $Q \rightarrow S$, but we should be careful interpreting this as we find a nonsmooth convergence. Moreover, the prediction of the location of the transition differs from other methods. All of this is to be expected from the strong crossover. Figure 6 shows curves for $L \eta_{-}(L)$ on the line $K=0.60$ for different values of $L$.

The exponent $x_{o}$ does not suffer from crossover as it is insensitive to Gaussian behavior. Convergence of this $x_{o}$ is smooth and the estimates do not vary over the line $Q-S$. We have very few points to determine this value because of our limited system sizes, but with smooth convergence we find $x_{o}=0.068(8)$. This value definitely differs from the Ising value $x=\frac{1}{8}$. The exponent pertaining to $\eta_{i}$ yields a value of $x_{i}=0.204(5)$ at point $K=0.60$, which is also inconsistent with Ising. Finally we determined the thermal exponent $x_{t}$. It is difficult to determine and exhibits generally a bad convergence. At $K=0.60$, however, the convergence shows to be good and yields $x_{t}=0.88(1)$. Its Ising value is $x_{t}=1$.

No exponent whatsoever is found consistent with Ising on the line $Q-S$.

\section{The line $S-U$}

On the line $S-U$, the KT line $R-S$ merges with the deconstruction line. We do not expect Ising exponents, however, as we did not find them on the line $Q-S$. Surprisingly, we find a (smoothly converging) central charge value of $c=1.47(1)$, which is close to its KT $\times$ Ising value, but seems to be even lower. However, central charges are notoriously difficult to calculate and the convergence could be an artifact of our small system sizes.

The Gaussian coupling $K_{g}$ does not display the universal behavior of Eq. (7), and its value at the transition seems to be lower than the universal value $\frac{1}{2} \pi$.

The exponent corresponding to $\eta_{-}$displays smooth convergence and yields a value of $x_{-}=0.200(2)$. The (admittedly inaccurate) determination of the exponent from $\eta_{o}$ gives $x_{o}=0.07(2)$. The exponent from $\eta_{i}$ displays nonsmooth convergence and gives $x_{i}=0.15(1)$, but the estimate of the error may be much too small. The thermal exponent shows for larger $W$ bad convergence and is impossible to determine. Just beyond point $S$, however, determination is still possible and we find $x_{t}=0.73(3)$ at $W=1.60$ and $x_{t}=0.72(7)$ at $W=1.75$. Beyond this point, $x_{t}$ seems to decrease, but no conclusions as to its value can be inferred from our data. We cannot even exclude the possibility of the transition becoming first order further away from point $S$.

\section{DISCUSSION}

Interpretation of our data apart from the lines $Q-S$ and $S-U$ is straightforward. The line $T-R-S$ is a KT line, $Q-R$ is a preroughening line, and $Q-P$ is a first order line, actually extending to the $K=0$ axis.

The line $Q-S$ is expected to be an Ising line but does not display Ising exponents. This could be due to the fact that it is squeezed between the two multicritical points $S$ and $Q$. Indeed in the clock step model of Den Nijs ${ }^{5}$ where the multicritical point $Q$ is absent, Ising-like behavior is found. On the other hand, in the model studied by Mazzeo et al. ${ }^{9}$ the phase diagram is in this respect similar to that of the clock step model, but these authors do find exponents deviating from Ising. ${ }^{10}$

A feature which is present in both our model and that of Mazzeo et al. but not in the clock step model is the presence of the vertices 5 and 6 (Fig. 1), to which no Ising spins are assigned. These vertices act like vacancies with a fugacity $1 / W$. It is quite conceivable that it is these vacancies that alter the universality class. The model displaying these vacancies is the tricritical Ising or Blume-Capel model. ${ }^{30}$ The model shows a critical (Ising) line terminating in a tricritical point beyond which the transition becomes first order.

The central charge of the tricritical Ising point is $c=\frac{7}{10}$ and its exponents are $x=\frac{3}{40}, \frac{1}{5}, \frac{7}{8}$, and $\frac{6}{5} .{ }^{31}$ Apart from the central charge, which shows a notorious bad convergence, we are able to identify the three most relevant exponents $x$. The exponent $\frac{3}{40}(0.075)$ is identified with $x_{o}=0.068(8)$ on $Q-S$ and (with larger error) with $x_{o}=0.07(2)$ on $S$ - $U$. The exponent $\frac{1}{5}(0.20)$ corresponds to $x_{i}=0.204$ (5) on $Q-S$, but not on $S-U$, where $x_{i}=0.15(1)$. The third exponent $x=\frac{7}{8}$ $(0.875)$ is found as $x_{t}=0.88(1)$ on the line $Q-S$. The last exponent $x_{-}$that we measured along $Q-S$ stems from antiperiodic BC's and suffers from strong crossover to Gaussian behavior. In the Gaussian phase, this exponent is $x=\frac{1}{8}$ as follows from Eq. (8). If the crossover is perfect, one tends to think that this value adds up to the actual value, which means that the exponent should be identified with $\frac{1}{5}-\frac{1}{8}$ yielding (coincidentally) $\frac{3}{40}$. Indeed, the finite size value rises up to about 0.20 and then starts to decrease for larger values of $L$. The exponent $x_{-}$should thus be identified with $\frac{3}{40}$. To complete the identification, the least relevant exponent $x=\frac{6}{5}$ should be sought for.

In summary, the line $Q-S$ shows tricritical Ising exponents within the error bars. On the line $S-U$, where the surface becomes rough as well, deviations from this behavior are found.

This coincidence could lead one to the conjecture that this part of the phase diagram is to be understood as a tricritical Ising model (coupled to a Gaussian model). However, apart from the fact that the tricritical Ising exponents are found along the whole line $Q-S$ and not in a single point, the puzzling feature is that the scaling fields to which these exponents belong do not fit. Our magnetic exponent $x_{i}$ is identified with the thermal tricritical exponent $x=\frac{1}{5}$ while our thermal exponent $x_{t}$ appears as the magnetic tricritical exponent $x=\frac{7}{8}$. Further research is needed to see whether the remarkable coincidence of the calculated exponents with the exponents of the tricritical Ising model is a mere accident or whether there is a deeper connection. A better understanding 
is even more called for in view of the large number of recent papers that discuss models with similar behavior.

Mazzeo, Levi, Jug, and Tosatti studied deconstruction and roughening of the $\mathrm{Au}(110)$ surface in a $\mathrm{MC}$ simulation. ${ }^{6}$ They find two separate but nearby transitions, and claim that the deconstruction transition is in the Ising universality class. Their result for the exponent

$$
\frac{\gamma}{\nu}=2-2 x
$$

where $x$ is a magnetic exponent, is $\gamma / \nu=1.8(2)$, which corresponds to an exponent $x=0.1(1)$, actually consistent with Ising as well as tricritical Ising behavior. The specific heat $C$ shows a logarithmic size dependence, indicating Ising-like behavior, but, as argued in Ref. 13, power law and logarithmic behavior may be very difficult to distinguish. We conclude therefore that their results do not necessarily indicate Ising behavior but are consistent with behavior deviating from Ising as well.

Mazzeo, Carlon, and Van Beijeren ${ }^{9}$ study the phase diagram of the two-component BCSOS model. They find a roughening transition initially separated from a deconstruction transition. The latter falls into the Ising universality class. When the two transitions become nearby, they find exponents deviating from Ising. They find a magnetic exponent $x_{o}$ well below the Ising value $\frac{1}{8}$ and a central charge above $\frac{3}{2} .10$

Another model showing an interplay between Gaussian and Ising degrees of freedom is the FF $X Y$ model. The model is believed to be equivalent with a line in the full phase diagram of the coupled $X Y$-Ising model, with Hamiltonian ${ }^{12,18}$

$$
H=-\sum_{\langle i, j\rangle} A\left(1+\sigma_{i} \sigma_{j}\right) \cos \left(\theta_{i}-\theta_{j}\right)+C \sigma_{i} \sigma_{j} .
$$

The angle variables $\theta$ are the $X Y$ variables and the $\sigma$ are Ising spins. The model shows an Ising line and a KT line, merging into a single critical line that eventually becomes first order. ${ }^{12,18}$ In studies of the FFXY model, most authors find that there is a single transition with exponents deviating from Ising. ${ }^{11,13,15,16}$ The hypothesis of the two transitions to be simultaneous is not always confirmed ${ }^{14}$ or is rejected. ${ }^{17} \mathrm{It}$ is thus believed that the FFXY model is located in the phase diagram of the coupled $X Y$-Ising model close to the merging of the Ising and KT lines. The single critical line of this model shows varying critical exponents, presumably due to crossover. The exponents found in the above mentioned papers display roughly the same values as in our model on the line $S-U$. The thermal exponent $x_{t}$ is generally found somewhat lower than the tricritical Ising value $\frac{7}{8}$, the exponent pertaining to $\eta_{-}$agrees fairly with $x=\frac{1}{5}$, and the correlation function exponent $x_{i}$ is about 0.15 .

We therefore conjecture that the joined transitions of the two-component BCSOS model, our model, and the coupled $X Y$-Ising model fall into the same universality class. We find varying critical exponents along this line, and the transition may eventually become first order, as in the coupled $X Y$ Ising model.

\section{CONCLUSIONS}

We have calculated the phase diagram of a singlecomponent BCSOS model with nearest and next-nearestneighbor interactions between atoms of each of the two sublattices, using transfer matrix techniques. We found a rich phase diagram, with flat, $2 \times 2$ reconstructed, DOF, and rough phases. Existence and character of the preroughening transition between flat and DOF phases as established by Den $\mathrm{Nijs}^{3,4}$ are confirmed by our calculations. The Ising-like deconstruction transition between the reconstructed and DOF phases actually shows exponents deviating from Ising. Merging of this line with a KT transition line gives rise to a simultaneous roughening and deconstruction transition with exponents deviating from Ising.

We stress the similarity of this interplay between roughening and Ising degrees of freedom with that in fully frustrated $X Y$ models, note that the observed exponents in both cases roughly coincide, and therefore argue that both transitions fall in the same universality class.

We observe qualitatively that the interplay between roughening and Ising degrees of freedom in our model may result in the effective appearance of Ising vacancies in the model, and compare our calculated critical exponents with those of the tricritical Ising point present in the phase diagram of the Blume-Capel model. We observe a remarkable coincidence, and conjecture that tricritical Ising behavior rather than Ising behavior may well be involved.

\section{ACKNOWLEDGMENTS}

It is a pleasure to thank Enrico Carlon and Henk van Beijeren for stimulating discussions.
*Electronic address: paulb@tvs.kun.nl

${ }^{1}$ J. Villain and I. Vilfan, Surf. Sci. 199, 165 (1988).

${ }^{2}$ I. Vilfan and J. Villain, Surf. Sci. 257, 368 (1991).

${ }^{3}$ Koos Rommelse and Marcel den Nijs, Phys. Rev. Lett. 59, 2578 (1987); Marcel den Nijs and Koos Rommelse, Phys. Rev. B 40, 4709 (1989).

${ }^{4}$ Marcel den Nijs, Phys. Rev. Lett. 64, 435 (1990).

${ }^{5}$ Marcel den Nijs, Phys. Rev. Lett. 66, 907 (1991); Phys. Rev. B 46, 10386 (1992).

${ }^{6}$ Giorgio Mazzeo, Giancarlo Jug, Andrea C. Levi, and Erio Tosatti, Surf. Sci. 273, 237 (1992).
${ }^{7}$ Giorgio Mazzeo, Giancarlo Jug, Andrea C. Levi, and Erio Tosatti, Phys. Rev. B 49, 7625 (1994).

${ }^{8}$ Marco Bernasconi and Erio Tosatti, Surf. Sci. Rep. 17, 363 (1993).

${ }^{9}$ Giorgio Mazzeo, Enrico Carlon, and Henk van Beijeren, Phys. Rev. Lett. 74, 1391 (1995).

${ }^{10}$ Enrico Carlon (private communication).

${ }^{11}$ J. M. Thijssen and H. J. F. Knops, Phys. Rev. B 42, 2438 (1990).

${ }^{12}$ Enzo Granato, J. M. Kosterlitz, Jooyoung Lee, and M. P. Nightingale, Phys. Rev. Lett. 66, 1090 (1991); Jooyoung Lee, Enzo Granato, and J. M. Kosterlitz, Phys. Rev. B 44, 4819 (1991). 
${ }^{13}$ Jooyoung Lee, J. M. Kosterlitz, and Enzo Granato, Phys. Rev. B 43, 11531 (1991).

${ }^{14}$ Guillermo Ramirez-Santiago and Jorge V. José, Phys. Rev. Lett. 68, 1224 (1992); G. Ramirez-Santiago and Jorge V. José, Phys. Rev. B 49, 9567 (1994).

${ }^{15}$ Enzo Granato and M. P. Nightingale, Phys. Rev. B 48, 7438 (1993).

${ }^{16}$ Y. M. M. Knops, B. Nienhuis, H. J. F. Knops, and H. W. J. Blöte, Phys. Rev. B 50, 1061 (1994).

${ }^{17}$ Peter Olsson, Phys. Rev. Lett. 75, 2758 (1995).

${ }^{18}$ M. P. Nightingale, E. Granato, and J. M. Kosterlitz, Phys. Rev. B 52, 7402 (1995).

${ }^{19}$ Paul J. M. Bastiaansen and Hubert J. F. Knops (unpublished).

${ }^{20}$ F. Rys, Helv. Phys. Acta 36, 537 (1963).

${ }^{21}$ E. H. Lieb, Phys. Rev. Lett. 18, 1046 (1967).

${ }^{22}$ H. van Beijeren, Phys. Rev. Lett. 38, 993 (1977).

${ }^{23}$ Erik Luijten, Henk van Beijeren, and Henk W. J. Blöte, Phys.Rev. Lett. 73, 456 (1994).

${ }^{24}$ See, e.g., P. Nightingale, J. Appl. Phys. 53, 7927 (1982).

${ }^{25}$ John L. Cardy, J. Phys. A 17, L385 (1984).

${ }^{26}$ H. W. J. Blöte, John L. Cardy, and M. P. Nightingale, Phys. Rev. Lett. 56, 742 (1986).

${ }^{27}$ A. P. Young, Phys. Rev. B 19, 1855 (1979).

${ }^{28}$ C. Itzykson and J. B. Zuber, Nucl. Phys. B 275, 580 (1986); for a concise derivation see Ref. 16.

${ }^{29}$ Leo P. Kadanoff and Horacio Ceva, Phys. Rev. B 3, 3918 (1971).

${ }^{30}$ M. Blume, Phys Rev. 141, 517 (1966); H. W. Capel, Physica (Utrecht) 32, 966 (1966); 33, 295 (1967); 37, 423 (1967).

${ }^{31}$ B. Nienhuis, J. Phys. A 15, 199 (1982); Marcel den Nijs, Phys. Rev. B 27, 1674 (1983); Vincent Pasquier, J. Phys. A 20, L1229 (1987). 